\title{
EL DERECHO PÚBLICO EN LA DETERMINACIÓN DEL INTERÉS SUPERIOR DE MENORES TERMINALES EN EL REINO UNIDO
}

\author{
ALEJANDRA BOTO ÁLVAREZ \\ Universidad de Oviedo \\ Profesora titular de Derecho Administrativo
}

Cómo citar/Citation

Boto Álvarez, A. (2020).

El derecho público en la determinación del interés superior de menores terminales en el Reino Unido. Revista de Administración Pública, 211 1, 161-184. doi: https://doi.org/10.18042/cepc/rap.211.06

\section{Resumen}

Se comentan tres casos recientes ocurridos en el Reino Unido que versan sobre las vías judiciales abiertas ante el desacuerdo entre familias y equipos médicos sobre el mantenimiento del soporte vital artificial a menores terminales. Tradicionalmente estos asuntos se reconducen al derecho privado, enfrentando el interés del menor con el parental. Sin embargo, también existen implicaciones de derecho administrativo, derechos humanos y derecho de la Unión. Se presta atención a la consideración de las decisiones médicas en el marco de los servicios públicos sanitarios como actos administrativos, la afección a la libertad de circulación y libre prestación de servicios, las implicaciones religiosas y el ejercicio de la discrecionalidad en el marco de la concesión de nacionalidad por carta de naturaleza.

\section{Palabras clave}

Interés superior del menor; control judicial; discrecionalidad administrativa; concesión de ciudadanía; encarnizamiento terapéutico; pediatría. 


\section{Abstract}

This paper comments three recent cases related to British and European Courts decisions on disputes regarding the withdrawal of life sustaining treatment to children. Those issues are usually considered under private law, confronting the best interest of the child with parental responsibility. However, there is also a public law perspective to explore, involving administrative law, human rights and EU citizenship. Special attention is paid to medical decisions made within the National Health Services as administrative action submitted to judicial review; interferences with free movement, provision and receipt of services; religious dimension and State discretion in the conferral of citizenship by naturalization.

\section{Keywords}

Best interest of the child; Judicial review; Discretionary powers; Citizenship conferral; Medical futility; Paediatrics. 


\section{SUMARIO}

I. INTRODUCCIÓN. II. LOS HECHOS Y EL DERECHO APLICABLE. III. ORDEN DE FACTORES Y PRODUCTO: LAS DECISIONES MÉDICAS COMO ACTOS ADMINISTRATIVOS SUSCEPTIBLES DE RECURSO AUTÓNOMO. IV. ARGUMENTOS DESCARTADOS: DERECHO A LA VIDA (TAMBIÉN PRIVADA Y FAMILIAR), DISCRIMINACIÓN Y VULNERACIÓN DE LA LIBERTAD DE CIRCULACIÓN Y MOVIMIENTO. V. LA DERIVA CONFESIONAL Y LAS INTROMISIONES INTERNACIONALES. VI. DE NUEVO SOBRE EL ORDEN DE FACTORES: LA LIBRE PRESTACIÓN DE SERVICIOS EN LA UE. VII. EL INTERÉS SUPERIOR DEL MENOR Y CUESTIONES FINALES.

\section{INTRODUCCIÓN ${ }^{1}$}

De forma muy reciente se han conocido a través de los medios de comunicación y las redes sociales distintas batallas legales acontecidas en el Reino Unido que tenían como telón de fondo una radical discrepancia en la manera de entender la continuidad del tratamiento para bebés irreversiblemente enfermos entre sus familias y los equipos médicos del servicio sanitario británico. Me refiero, fundamentalmente, a los casos de Charlie Gard (2017), Alfie Evans (2018) y Tafida Raqeeb (2019). Los casos Gard y Evans fueron contestados en distintas sedes de la jerarquía jurisdiccional doméstica y llegaron, en el caso de Evans, incluso hasta en varias ocasiones, ante el TEDH. Ambos lances han tenido lamentablemente un resultado fatal con la muerte de los menores. En el momento de escribir estas páginas, Raqeeb sigue viva y, tras un fallo de la High Court cuyo análisis ocupará el grueso del presente trabajo, ha sido trasladada a un hospital italiano, solución que infructuosamente también intentaron en su momento los padres de Evans.

1 El presente trabajo ha sido elaborado gracias a una estancia financiada por el proyecto de investigación de referencia IDI/2018/000219 correspondiente a una ayuda a organismos públicos de investigación para apoyar las actividades de grupos de investigación en el Principado de Asturias concedida por el Gobierno de este al grupo SPAG (Servicios Públicos, Administraciones y Garantías), que coordina el profesor L. Tolivar Alas en la Universidad de Oviedo. 
Los tres casos tienen en común el haber atraído una atención global sin precedentes, que ha dado pie a variopintas declaraciones oficiales e institucionales de procedencias diversas, y no siempre directamente relacionadas con el contexto en discusión. No es de extrañar, pues se ponen de manifiesto cuestiones polémicas no solo desde la perspectiva médica y jurídica, sino también de carácter ético, social y religioso.

El desacuerdo entre familias y facultativos sobre la continuidad de tratamientos médicos en el caso de menores terminales no es algo novedoso y, como es lógico, la atención de la doctrina jurídica hacia ello, tampoco ${ }^{2}$. No obstante, en general los estudios del tema se han enfocado desde una perspectiva sectorial (médica ${ }^{3}$ o bioética $\left.{ }^{4}\right)$, procesal (con el reclamo de un ámbito para la mediación $\left.{ }^{5}\right)$ o iusprivatista (por las implicaciones para el derecho de familia y la patria potes$\operatorname{tad}^{6}$ ). Evidentemente la cuestión también tiene connotaciones constitucionales, que normalmente, sin embargo, han sido tratadas de forma incidental, bien centrándose sobre el fenómeno objetivo (la muerte ${ }^{7}$ ) o subjetivo (el menor $\left.{ }^{8}\right)$, y con cierta atención al conflicto con la libertad religiosa, no siempre en el ámbito de la medicina paliativa, sino también curativa9. Lo que aquí se trata de mostrar es que la cuestión también se puede declinar en clave de instituciones generales del derecho administrativo, derechos humanos y derecho de la Unión ${ }^{10}$. En efecto, decisiones discrecionales, motivación e impugnación de los actos, no discrimi-

2 Entre los primeros ejemplos véase M. Brazier (2005), «An Intractable Dispute: When Parents and Professionals Disagree», Medical Law Review, 13:3, págs. 412-418.

3 Por todos, entre nosotros, M. V. García-Atance García de Mora (2016), «Dignidad de la persona en el proceso de morir y muerte digna: especial referencia al menor», Derecho y salud, 26, 1, págs. 169-179.

4 Así, por ejemplo, puede verse el monográfico dedicado al caso Evans en el núm. 2 (2018) de la revista BioLaw Journal-Revista di BioDiritto. Sobre el caso Gard, véase L. De Panfilis (2018), "Il caso di Charlie Gard tra condivisione delle scelte di cura e processi decisionali etici», Revista de bioética y derecho: publicación del Máster en bioética y derecho, 43, págs. 277-289.

5 Por todos, G. Birchley (2014), «Deciding Together? Best Interests and Shared Decision-Making in Paediatric Intensive Care», Health Care Analysis, 22:3, págs. 203-222.

6 Sirvan como ejemplo los trabajos recogidos en el libro colectivo, editado en 2017, S. Sanz Caballero (dir.), El interés superior del niño en la jurisprudencia internacional, comparada y española, Valencia: Tirant lo Blanch.

7 R. Chueca (2009), «El marco constitucional del final de la propia vida», Revista de Derecho Constitucional, 85, págs. 99-123.

8 Entre los estudios más concretos merece la pena citar el de B. Aláez Corral (2003), Minoría de edad y derechos fundamentales, Madrid: Tecnos.

9 Así, R. Corral García (2004), «STC 154/2002: la negativa a una transfusión de sangre a un menor con el resultado de su muerte», Anuario da Facultade de Dereito, 8, págs. 987-993.

10 Sin olvidar que en todo caso la cuestión rebasa los artificiosos moldes y las ficticias fronteras entre los distintos sectores del ordenamiento jurídico, tal y como manifestara en su 
nación, libertad de movimiento y libre prestación de servicios en el territorio de la Unión tienen mucho que decir en la solución concreta que se ha dado a cada caso. En los tiempos inciertos del brexit, casi huelga mencionarlo, el análisis de la incidencia de estas últimas cuestiones queda reforzado.

El trabajo comienza con una sucinta introducción a los tres casos de referencia ${ }^{11}$ y la presentación del marco jurídico aplicable a este tipo de controversias en el Reino Unido, para pasar después a centrarse en los aspectos más relevantes desde la perspectiva comentada del derecho público.

\section{LOS HECHOS Y EL DERECHO APLICABLE}

Charlie Gard y Alfie Evans fueron dos bebés británicos que ante enfermedades muy graves recibieron tratamiento en renombrados hospitales públicos británicos, en concreto en el Great Ormond Street Hospital for Children NHS Foundation Trust de Londres, y en el Aldey Hey Children's NHS Foundation Trust de Liverpool, respectivamente. Su situación captó la atención pública cuando los padres, primerizos y muy jóvenes en los dos supuestos, manifestaron su oposición radical a la decisión médica de retirar el soporte vital que mantenía con vida a sus hijos. En ambos expedientes la decisión médica fue validada por el Tribunal Superior (High Court of Justice) y tras apelaciones frustradas, también ambas familias recurrieron, sin éxito, ante el TEDH.

Los padres de Gard tenían la esperanza de que su hijo, un bebé con apenas seis meses, pudiera acceder a un tratamiento experimental en Estados Unidos, emprendiendo incluso un mecanismo de economía colaborativa para sufragarlo ${ }^{12}$; el equipo médico y ulteriormente también la justicia entendieron, en cambio, que esa sería una alternativa fútil, contraria al interés del menor. El caso fue juzgado en el Tribunal Superior en primera instancia por Justice Francis ${ }^{13}$, cuyo parecer sería confirmado por la Corte de Apelación ${ }^{14}$. Los padres quisieron recurrir esta última decisión ante la Corte Suprema, pero su pretensión no fue admitida ${ }^{15}$; llegaron ante el TEDH, que también declaró inadmisible el recurso ${ }^{16}$. Finalmente, el caso

momento L. Tolivar Alas (1991), "Aspectos jurídico-administrativos de la protección de menores", Revista de Administración Pública, 124, págs. 35-64.

11 El relato se extrae de los antecedentes de hecho contenidos en los distintos fallos judiciales que serán analizados. Se evitan intencionadamente los reportes periodísticos, por lo sensible del tema y la dificultad de mantener una aproximación objetiva a los casos.

12 Parece que llegaron a recaudar cerca de un millón trescientas mil libras esterlinas: C. Dyer (2017), «Law, Ethics, and Emotion: The Charlie Gard Case», British Medical Journal, 358.

13 Great Ormond Street Hospital v Yates and others [2017] EWHC 972 (Fam).

14 Yates \& Gard $v$ Great Ormond Street Hospital \& Gard [2017] EWCA Civ 410.

15 In the Matter of Charlie Gard (Supreme Court, 8th June 2017).

16 Gard and Others $v$ United Kingdom (application number 39793/17). 
volvió ante el Tribunal Superior para la ejecución de la orden de desconexión ${ }^{17}$, los padres no formularon ya más oposición y el bebé moría unos días más tarde.

Los padres de Evans, por su parte, pretendieron un traslado del bebé, que por entonces tenía un año y medio, a Italia con el aval de la concesión por carta de naturaleza de esa nacionalidad —algo que, sin embargo, nunca llegó a verificarse, como luego se verá-. El centro elegido para continuar el tratamiento era el Ospedale Pediatrico Bambino Gesù, perteneciente al servicio público sanitario italiano, pero con fuertes vinculaciones con la Santa Sede. Esto, junto a algunas declaraciones del papa Francisco en redes sociales y al hecho de que los padres profesaran la religión católica, hizo que el debate adquiriera cierta deriva confesional. El hospital italiano había anunciado públicamente que se haría cargo de mantener el soporte vital a Evans de manera gratuita y por razones «humanitarias», lo que pudo haber supuesto otro frente de crisis por atentar contra principios bien conocidos del derecho internacional público, como el de no intervención en asuntos domésticos y la nacionalidad efectiva basada en vínculos genuinos. Procesalmente el caso es aún más imbricado que el de Gard. Fue juzgado en primera instancia por el Tribunal Superior ante Justice Hayden, quien decretó que mantener al bebé con ventilación artificial no respondía a su mejor interés ${ }^{18}$, no siendo tampoco recomendable el viaje a Italia porque no mejoraría su situación y, al contrario, corría el riesgo de ponerle en mayor riesgo ${ }^{19}$. El parecer sería confirmado por la Corte de Apelación ${ }^{20}$ y por la Corte Suprema ${ }^{21}$, y el posterior intento de recurso ante el TEDH inadmitido ${ }^{22}$. El caso volvió ante el Tribunal Superior para ejecución y ante el plan de desconexión planificado por el hospital, los padres presentaron un habeas corpus que fue denegado por el mismo juez Hayden ${ }^{23}$. Esta nueva decisión fue recurrida,

17 Great Ormond Street Hospital v Yates, Gard \& Gard [2017] EWHC 1909 (Fam).

18 La expresión inglesa «best interest of the child» suele traducirse como «interés superior del menor». En este trabajo se juega también con su traducción literal («mejor interés del menor») para indicar no solo la superioridad del interés del niño frente al de sus padres, sino para evocar también que se trata de decidir en el fondo «lo mejor» para aquel. Incidentalmente también cabe señalar que los tres casos aquí comentados se refieren a niños muy pequeños, con graves limitaciones físicas y sin madurez (capacidad o incluso consciencia) suficiente para ser consultados sobre la continuidad de su soporte vital. Por razones de espacio no se reproducen con detalle los datos de las enfermedades que padecían y los distintos diagnósticos respecto a su carácter permanente o terminal.

19 Aldey Hey Children's NHS Foundation Trust v Evans, James \& Evans [2018] EWHC 308 (Fam).

$20 \operatorname{Re} E$ (A Child) [2018] EWCA Civ 550.

21 In the Matter of Alfie Evans (20th March 2018).

22 Evans $v$ United Kingdom (application no. 14238/18).

23 Aldey Hey Children's NHS Foundation Trust v Evans, James \& Evans [2018] EWHC 818 (Fam). 
sin éxito, ante la Corte de Apelación ${ }^{24}$ y la Corte Suprema ${ }^{25}$. El subsiguiente intento de recurso ante el TEDH fue también inadmitido ${ }^{26}$. El bebé fue desconectado, pero continuó viviendo, ante lo que los padres presentaron una nueva acción frente al Tribunal Superior para trasladar al bebé a Italia; la petición fue desestimada, por no apreciarse cambios respecto a la situación juzgada de Evans $^{27}$. Frente a esta decisión los padres recurrieron una vez más de manera infructuosa ante la Corte de Apelación ${ }^{28}$ y su hijo moriría tres días más tarde.

Por su parte, el recorrido hospitalario de Tafida Raqeed se produjo asimismo en prestigiosos centros públicos del Reino Unido: con cuatro años de edad, en febrero de 2019, fue operada de urgencia en el Newham University Hospital tras la espontánea ruptura de una malformación arteriovenosa, y posteriormente trasladada con importantes secuelas cerebrales, primero al Kings College Hospital, y después al Royal London Hospital. Con posiciones irreconciliables sobre el mantenimiento del soporte vital artificial entre la familia y el equipo médico, el 16 de julio de 2019 el Barts Health NHS Trust (gestor de este último centro) accionaba ante el Tribunal Superior para conseguir la orden de su retirada; con esa misma fecha los padres interponían recurso contra la decisión administrativa del Trust negándose al traslado de la pequeña a Italia, donde también existía un hospital dispuesto a continuar su tratamiento (el Istituto Giannina Gaslini). Esta vez no existía, sin embargo, ninguna oferta de gratuidad, ni tampoco manifestaciones institucionales desde el Gobierno italiano. La familia sí contó con un dictamen jurídico de parte emitido por el doctor Giacomo Rocchi, magistrado de la Corte Suprema de Casación italiana, que fue admitido por el tribunal. Hubo también aportaciones del Alto Comisionado de Bangladesh en el Reino Unido, dado que los padres de Raqeed tienen también esa nacionalidad. Puede verse como el aspecto internacionalista sigue presente, así como el religioso. Raqeed y su familia profesan el islam, y en su caso ante el Tribunal presentaron un dictamen (en forma de fatwa) emitido por el Islamic Council of Europe. En una sentencia particularmente larga, Justice Macdonald acabaría desestimando la petición del Trust para la desconexión del soporte vital, lo que tiene como efecto el mantenimiento de la nińa con vida ${ }^{29}$.

24 Evans \& James v Alder Hey Children's NHS Foundation Trust \& Alfie Evans [2018] EWCA Civ 805.

25 In the Matter of Alfie Evans No. 2 (20th April 2018).

26 Evans $v$ the United Kingdom (application no. 18770/18).

27 Alder Hey Children's NHS Foundation Trust v Evans, James \& Evans [2018] EWHC 953 (Fam).

28 Evans \& James v Aldey Hey Children's NHS Foundation Trust \& Evans [2018] EWCA 984 (Civ).

29 Barts Health NHS Trust v Raqeeb [2019] EWHC 2531 (Admin) and [2019] EWHC 2530 (Fam). 
Aunque con distinta suerte, el recorrido procesal en todos los casos fue similar. En principio, la legislación británica (Children Act 1989) reconoce a los padres el derecho a decidir sobre el tratamiento que reciben sus hijos, pero no se trata de un derecho absoluto. Cuando una autoridad sanitaria considere que la decisión parental no responde al interés superior del menor, y el acuerdo entre ambas partes no sea posible, la decisión final será tomada por un juez, en un procedimiento contradictorio y fundándose en una evaluación objetiva del mejor interés del menor. Distinta jurisprudencia ha sentado que el juez asume entonces un rol parens patriae y aunque los padres no quedan del todo excluidos en la toma de la decisión, porque existe un sistema de checks and balances, la decisión es responsabilidad judicial ${ }^{30}$. Ni la familia ni las autoridades sanitarias pueden imponer su parecer unilateralmente, sino que tienen la obligación de instar un procedimiento judicial para que sea el juez quien se pronuncie. De conformidad con el art. 8 del Reglamento Bruselas II.bis ${ }^{31}$, la competencia recae en los tribunales del lugar de residencia habitual del menor. La acción judicial puede ser iniciada por el equipo médico que trata al paciente o por los padres, y ha de plantearse siempre que exista una discrepancia irresoluble ${ }^{32}$.

El elemento crucial en el juicio es el interés superior del menor, entendido en sentido amplio y teniendo en cuenta no solo su bienestar médico, sino también social y psicológico ${ }^{33}$. Ni la inviolabilidad de la vida ${ }^{34}$ ni la voluntad parental $^{35}$ pueden imponerse a ese interés, que, sin embargo, es algo a veces muy difuso y jurídicamente difícil de determinar ${ }^{36}$. Existen así voces críticas en la doctrina que denuncian un excesivo peso de la interpretación clínica sobre el

30 Ideas tomadas de las palabras de lord Donaldson MR en el caso Re J (a minor) (wardship: medical treatment) [1990] 3 All ER 930, par. 934, [1991] Fam 33, par. 41.

31 Reglamento (CE) 2201/2003: competencia, reconocimiento y ejecución de resoluciones judiciales en materia matrimonial y de responsabilidad parental.

32 Expresión de lady Black en An NHS Trust v Y [2018] UKSC 46, par. 109.

33 Palabras de la baronesa Hale en Aintree University Hospital NHS Trust v James [2013] UKSC 67, par. 39.

34 Existe, sin embargo, una presunción importante en favor de la conservación de la vida, por entender que es natural el instinto humano de supervivencia: Airedale NHS Trust $v$ Bland [1993] ACR 789, par. 825. Con todo, no es una presunción absoluta y cabe modularla en función de la calidad de vida, por ejemplo.

35 En el juicio no debe entrarse a valorar la razonabilidad de los argumentos parentales: Waite LJ, en Re T (A Minor) (Wardship: Medical Treatment) [1997] 1 All ER 906, par. 916-917. Pero sí deben tenerse en cuenta los derechos de los padres, por ejemplo en materia de libertad religiosa: Justice Macdonald en Barts Health NHS Trust v Raqeeb [2019] EWHC 2531 (Admin) and [2019] EWHC 2530 (Fam), par. 116.ix)

36 J. Bridgeman (2007), Parental Responsibility: Young Children and Health Care, Cambridge: Cambridge University Press (pág. 103). 
interés del menor, a falta de suficiente expertise para juzgarlo en el tribunal ${ }^{37}$. Según estas opiniones, sería más adecuado sustituir el test del «mejor interés del menor» por el del «daño significativo»; así, la voluntad de los padres debería seguirse salvo que se probara que ello supondría un daño intolerable para el menor ${ }^{38}$.

La propuesta no es baladí porque la referencia al daño significativo está también presente en la Children Act 1989. El paradigma del daño para el menor es operativo en la perspectiva iuspublicista de protección de menores por parte de las autoridades locales británicas, de suerte que estas solo intervienen tomando a los niños a su cargo cuando la tutela de los progenitores está dañando al niño, como una intervención de ultima ratio ${ }^{39}$; pero estas autoridades locales no suelen intervenir cuando la disputa es clínica, porque esos casos se mueven en principio en un ámbito privado, donde ante el conflicto entre la visión de los médicos y la de los padres, se busca una solución judicial en el ámbito civil ${ }^{40}$. Están configurados como supuestos distintos, pero teniendo en cuenta el carácter administrativo del servicio sanitario público (NHS) ${ }^{41}$, no parece del todo descabellado pensar que los ámbitos puedan entrecruzarse ${ }^{42}$.

37 R. Heywood (2012), «Parents and Medical Professionals: Conflict, Cooperation and Best Interests», Medical Law Review, 20:1, págs. 29 y ss.

38 Por todos, D. S. Diekema (2004), «Parental Refusals of Medical Treatment: The Harm Principle as Threshold for State Intervention», Theoretical Medicine and Bioethics, 25, págs. 243-264.

39 La idea fue así formulada por la Comisión que informó la norma en su momento, entendiendo en su epígrafe 2.1 que «[...] the primary responsibility for the upbringing of children rests with their parents. The State should be ready to help them to discharge that responsibility and should intervene compulsorily only where the child is placed at unacceptable risk». El texto completo del informe puede consultarse en https://s3-eu-west-2. amazonaws.com/lawcom-prod-storage-11 jsxou24uy7q/uploads/2016/07/LC.-172-FAMILY-LAW-REVIEW-OF-CHILD-LAW-GUARDIANSHIP-AND-CUSTODY.pdf.

40 Los supuestos se corresponden con dos incisos distintos de la Children Act 1989. Uno se enmarca dentro de la sección 31 (2) que dispone que: «[...] A court may only make a care order or supervision order if it is satisfied - (a)that the child concerned is suffering, or is likely to suffer, significant harm; and (b)that the harm, or likelihood of harm, is attributable to-(i)the care given to the child, or likely to be given to him if the order were not made, not being what it would be reasonable to expect a parent to give to him; or (ii) the child's being beyond parental control». El otro se corresponde con la sección 3 (5), que reza: «[...] A person who-(a)does not have parental responsibility for a particular child; but (b)has care of the child, may (subject to the provisions of this Act) do what is reasonable in all the circumstances of the case for the purpose of safeguarding or promoting the child's welfare».

41 Tal y como señaló el juez Francis en su segunda sentencia sobre el caso Gard (citada en la nota 17, par. 17): To most like-minded people, a National Health Service trust is as much an arm of the state as is a local authority.

42 G. Birchley (2019), "The harm threshold: A view from the clinic», en I. Goold, J. Herring y C. Auckland (eds.), Parental Rights, Best Interests and Significant Harms (págs. 107-135), Oxford: Hart Publishing (en particular a la pág. 111). 
El criterio del daño, con todo, está lejos de ser pacífico, y la regulación positiva sigue pivotando en torno al concepto del interés del menor, a cuya búsqueda tiene que atenerse el tribunal ${ }^{43}$. En la doctrina se ha insistido en que, aunque esté presente un hospital público, la determinación del mejor interés para el menor en caso de discrepancia entre padres y médicos es una cuestión de derecho privado y por tanto no es aplicable el paradigma de intervención del derecho público: el daño significativo ${ }^{44}$. Sin negar la conclusión, porque la analogía entre la tutela administrativa del menor y la decisión sobre su futuro vital es errónea, tanto en su base legal ${ }^{45}$ como sobre todo en su causa ${ }^{46}$, el planteamiento de una separación plena público-privada es reduccionista, como se verá a continuación.

La evolución jurisprudencial indica que las decisiones siempre tienen que descender a lo concreto, que en ocasiones hay inconsistencias y que no es sencillo generalizar las pautas para identificar el mejor interés del menor terminal, sobre todo cuando este es de cortísima edad ${ }^{47}$. De entre todos los recorridos procesales, el más corto y también el más peculiar es el del caso Raqeeb, donde no solo se planteaba la controversia sobre la desconexión en el marco de la Children Act 1989, sino que los padres contestaron judicialmente y de manera autónoma la negativa del hospital

43 Así, una cosa es retirar la tutela a los padres para dársela al Estado por un daño insoportable para el menor, y otra decidir sobre el mejor interés del niño en los casos de enfermedades terminales, tal y como sentó lady Hale en su explicación del pronunciamiento de la Corte Suprema sobre el caso Gard (https://www.supremecourt.uk/news/permission-toappeal-hearing-in-the-matter-of-charlie-gard.html). De la misma forma, no es lo mismo determinar cuándo, por razones de «ingeniería social», el menor debe ser apartado de los padres, quizá de manera temporal, para colocarlo bajo la protección del Estado, y cuándo debe ser apartado del mundo en global para ser entregado a la muerte, tal y como se planteó la Corte Suprema en el caso Evans (https://www.supremecourt.uk/news/permission-to-appeal-determination-in-the-matter-of-alfie-evans.html). La cuestión se zanja en el caso Raqeeb, sentencia citada en la nota 29, par. 104.

44 J. Bridgeman (2018), «A threshold of significant harm (f)or a viable alternative therapeutic option?», Journal of Medical Ethics, 44, págs. 466-470.

45 Según los preceptos transcritos en la nota 40. Así también lo sentó la Corte de Apelación en su primer examen del caso Evans (sentencia citada en la nota 20, par. 111).

46 Como se ha visto, la puesta del menor bajo la responsabilidad de los servicios sociales locales se debe al cuidado insatisfactorio por los padres, mientras que en los casos que aquí se plantean los padres no eran negligentes en ese cuidado, ni mucho menos, simplemente mantenían una postura diferente a la médica sobre si debía mantenerse el soporte vital artificial. Al respecto puede verse W. Seagrim (2019), «Best Interests: The 'Gold Standard' or a Gold Plating? Should Significant Harm be a Threshold Criterion in Paediatric Cases?», en K. A. Choong (ed.), Medical Futility in Paediatrics: Interdisciplinary and International Perspectives (págs. 51 y ss.), Budapest: Trivent Publishing (en particular págs. 62-64).

47 J. Samanta (2019), «The Vexed Question of Best Interests in Decisions Relating to Infants and Medical Futility», en K. A. Choong (ed.), Medical Futility in Paediatrics: Interdisciplinary and International Perspectives (págs. 29-50), Budapest: Trivent Publishing. 
británico al traslado de su hija a Italia, por entender que se trataba de un acto administrativo susceptible de enjuiciamiento previo a cualquier cuestión ulterior en torno a los intereses de su hija. A continuación, se presta atención detallada a este argumento, para pasar seguidamente a analizar otros elementos en liza.

\section{ORDEN DE FACTORES Y PRODUCTO: LAS DECISIONES MÉDICAS COMO ACTOS ADMINISTRATIVOS SUSCEPTIBLES DE RECURSO AUTÓNOMO}

Mientras que en los casos Gard y Evans, la voluntad de los padres de trasladar a sus hijos a otros países para continuar su tratamiento se debatió de forma conjunta, y eventualmente posterior, a la determinación de su superior interés, en el caso Rabqeeb se plantearon dos tipos de cuestiones dentro del mismo proceso judicial. La diferente estrategia condujo a un resultado formalmente distinto, pues el juez del Tribunal Superior entendió que habría de atender dos casos: el número CO/2767/2019, por un judicial review frente a la decisión del Trust de no acceder al traslado de la niña a Italia, y el FD19P00378, en aplicación de la Children Act 1989, para determinar si el superior interés de la misma era la retirada del soporte vital artificial que defendía el Trust frente a la opinión contraria de la familia ${ }^{48}$.

En el procedimiento en materia administrativa la nińa actúa como demandante a través de un representante que integra su falta de capacidad procesal (litigation friend) y asistida de abogados propios. El Trust es la parte demandada y los padres intervienen como interesados. En el procedimiento en aplicación de la Children Act 1989, el Trust es la parte procesal activa al pedir la orden de desconexión del soporte vital; los padres actúan oponiéndose a esa pretensión y la niña es parte, representada por un tutor legal (Children's guardian).

La argumentación básica de la familia es que antes de analizar cualquier aspecto relacionado con el interés de su hija, esta tiene derecho a un anterior procedural ruling respecto a la decisión del Trust de no acceder a su traslado a Italia. Y esto de acuerdo con la National Health Service Act 2006, y teniendo en cuenta que el Trust es una autoridad pública sometida a esa norma.

En la motivación del recurso se planteaba que el Trust no había tomado la decisión a partir de su entendimiento del mejor interés de la niña, sino por las diferencias éticas o filosóficas entre la regulación británica e italiana en la materia ${ }^{49}$, teniendo en cuenta la nacionalidad inglesa de la paciente. Además, consideraban que la decisión del Trust era ilegal por vulnerar los arts. 18, 21 y 56 del TFUE, así como los arts. 5, 8, 9 y 14 del CEDH. Volveremos sobre el fondo del asunto

48 Barts Health NHS Trust $v$ Raqeeb (sentencia completa citada en nota 29), pars. 3-6.

49 El margen de apreciación nacional en este tema concreto ha sido validado por el TEDH precisamente en el caso Gard (sentencia citada en la nota 16, pars. 84 y 122). 
más adelante, estudiando las diferencias y similitudes que plantea con respecto a los casos precedentes.

En su oposición a la demanda, el Trust sostenía que no había adoptado una decisión formal de oposición al traslado, sino que sencillamente había manifestado que esto no respondería al mejor interés de la paciente y, en su opinión, la decisión sobre el tema compete al tribunal en aplicación de la Children Act 1989. La única decisión formal del Trust fue, según su perspectiva, la de accionar ante la división de familia del tribunal para conseguir la orden de desconexión en el marco de la determinación judicial del superior interés del menor, y frente a ello no cabe recurso ante la división administrativa, sino seguir el procedimiento ordinario en la corte de familia. Su argumento principal es que no debe discutirse dónde puede prestarse el tratamiento médico, sino si tal tratamiento tiene sentido per se dentro del mejor interés de la niña ${ }^{50}$.

En la sentencia se entiende que la decisión del Trust, que efectivamente se oponía al traslado de la paciente, nada tenía que ver con su nacionalidad o con la diversidad de regulación nacional dentro de la UE, sino que se enmarcaba en su propio entendimiento del mejor interés de la menor, para cuya determinación se acude al tribunal ${ }^{51}$. Esto no significa, sin embargo, que tal decisión quede exenta de control judicial, y esto aunque exista pendiente otro procedimiento concurrente para la determinación del perímetro del mejor interés del menor ${ }^{52}$. En la medida en que los médicos eran empleados públicos resulta de aplicación la previsión de la NHS Constitution que señala literalmente que: «You have the right to make a claim for judicial review if you think you have been directly affected by an unlawful act or decision of an NHS body or local authority ${ }^{53}$. Por ello se admite el judicial review y se entra en el fondo del asunto, pues se trata de accionar contra una public authority, en relación con un asunto de derecho público y los actores cuentan con legitimación activa ${ }^{54}$.

El hecho de que en la toma de su decisión el Trust ni siquiera hubiera tenido en cuenta los derechos directamente efectivos de la niña en cuanto ciudadana de la Unión lleva al tribunal a entender que su decisión resulta prima facie ilegal ${ }^{55}$. En este punto el tribunal aplica la jurisprudencia sentada por la Corte de Apelación en el llamado caso Blood, según la cual una autoridad pública debe plan-

50 Enfasis en el original: Barts Health NHS Trust v Raqeeb, par. 58.

51 Ibid., par. 140.

52 Ibid., par. 141

53 El texto está disponible en https://www.gov.uk/government/publications/the-nhs-constitution-for-england/the-nhs-constitution-for-england.

54 De acuerdo con lo previsto en el Pre-Action Protocol for Judicial Review, disponible en https://www.justice.gov.uk/courts/procedure-rules/civil/protocol/prot_jrv. Sobre el judicial review en el Reino Unido, véase por todos A. W Bradley, K. D Ewing y C. Knight (2018), Constitutional and Administrative Law, 17a ed., London: Pearson, págs. 641 y ss.

55 Barts Health NHS Trust $v$ Raqeeb, par. 144. 
tearse si su decisión está vulnerando en primer lugar un derecho comunitario con efecto directo, y en segundo lugar estudiar si tal vulneración está justificada por ejemplo en razones de interés público ${ }^{56}$.

Siguiendo con ese mismo precedente, el tribunal continúa afirmando que, no obstante, de haber tenido el Trust en cuenta la afectación de esos derechos, su conclusión final hubiera sido que la interferencia estaba justificada, por entender que caía dentro de su interpretación del superior interés del menor ${ }^{57}$. Ante la evidente discrepancia con el parecer de los padres, el Trust inició el procedimiento para instar una decisión judicial, según una norma que no entra en conflicto con la primacía del derecho de la Unión, no es discriminatoria por razones de nacionalidad, resulta consistente con el Tratado, proporcionada y responde al interés público ${ }^{58}$. Procede entonces entrar en el fondo de esa cuestión, de acuerdo también con el propio derecho de la Unión, en concreto con el Reglamento Bruselas II.bis ${ }^{59}$.

Por tanto, señala el tribunal, el Trust no procedió correctamente, pero de haberlo hecho el asunto habría llegado exactamente al mismo punto ${ }^{60}$. Se produce entonces una pírrica victoria de la familia en este extremo, dado que en uso de la discrecionalidad conferida por el common law al tribunal, la parte ganadora del pleito no recibe en este caso ningún tipo de resarcimiento a pesar de haber quedado probado que en el proceder del Trust se produjo una vulneración de derechos directamente derivados de la Unión Europea ${ }^{61}$.

Estamos aquí ante una regla de common law que ha sido positivizada a través de la Criminal Justice and Courts Act 2015, que modificó a su vez la Senior Courts Act $1981^{62}$. El pronunciamiento es interesante en cuanto supone caracterizar la actividad del Trust como un acto administrativo susceptible de judicial review, y no como una simple actividad material frente a la que el ordenamiento no siempre garantiza a los ciudadanos la tutela judicial efectiva ${ }^{63}$. Y, sin embargo, el

56 Rv Human Fertilisation and Embryology Authority ex parte Blood [1999] Fam 151, [1997] 2 WLR 806, [1997] 2 FLR 742.

57 Barts Health NHS Trust $v$ Raqeeb, par. 146.

58 Ibid., pars. 148-151 y 154.

59 Ibid., pars. 152-153.

60 Ibid., par. 155.

61 Ibid., pars. 156-158.

62 En su redacción actual esta última dispone (s. 31) que: «The High Court (a)must refuse to grant relief on an application for judicial review, and (b)may not make an award under subsection (4) on such an application, if it appears to the court to be highly likely that the outcome for the applicant would not have been substantially different if the conduct complained of had not occurred».

63 Sobre el tema, en relación con la legislación española, puede verse A. Huergo Lora (2011), «Del recurso contra la vía de hecho al recurso contra la actividad material de la Administración», Revista General de Derecho Administrativo, 28. 
margen de la decisión judicial es tan amplio que el vencedor del recurso contencioso no obtiene ningún remedy: requerimiento, resarcimiento de daños, declaración, anulación, libramiento de orden ni prohibición ${ }^{64}$.

\section{ARGUMENTOS DESCARTADOS: DERECHO A LA VIDA (TAMBIÉN PRIVADA Y FAMILIAR), DISCRIMINACIÓN Y VULNERACIÓN DE LA LIBERTAD DE CIRCULACIÓN Y MOVIMIENTO}

Aunque a priori se trata de argumentos poderosos, en ninguno de los tres casos que aquí se toman como referencia tuvo viabilidad la invocación de estos derechos del menor o de los padres, ni ante los tribunales nacionales ni ante el TEDH.

En los casos Evans y Raqeed, la eventual discriminación (prohibida por los arts. 18 del TFUE y 14 del CEDH, entre otros) se trató de hacer valer a partir del elemento comparativo que supondrían otros nińos en otros Estados miembros (en concreto en Italia), donde sí estaría disponible el mantenimiento del soporte vital artificial para sus casos. En el caso Raqeed los padres argumentaban que el Trust no se habría opuesto al retorno a Italia de un bebé italiano que hubiera caído enfermo durante una estancia en el Reino Unido ${ }^{65}$. En el caso Gard, por su parte, se aportó al pleito el testimonio de un especialista norteamericano que afirmó que de haber estado el niño en Estados Unidos, habría sido tratado ${ }^{66}$. No obstante, el argumento cedió ante el del mejor interés del niño, pues incluso ese especialista reconocía que era muy improbable que el niño mejorara con el tratamiento alternativo experimental ${ }^{67}$.

El arquetipo del superior interés del menor es también el que se pondera frente al art. 2 del CEDH. Lo mismo ocurre respecto de la responsabilidad parental y la vida familiar, que repetidamente trató de hacer valerse a través del art. 8 del mismo cuerpo normativo.

El argumento de una posible discriminación por nacionalidad se analizará en el epígrafe siguiente, pues en el caso Evans se planteó incluso un cambio sobrevenido de esta. También en ese punto se tendrán en cuenta distintas invocaciones de la libertad religiosa.

Por otra parte, los padres de Raqeed sostuvieron también que el mantenimiento de la nińa en el hospital era contrario al art. 5 del CEDH porque no respondía al propósito de mantenerla viva, sino solo a evitar el viaje a Italia ${ }^{68}$.

\footnotetext{
64 Sobre los remedies en derecho británico puede verse M. Elliott y R. Thomas (2017), Public Law, $3^{\mathrm{a}}$ ed., Oxford: Oxford University Press, págs. 556-589.

65 Barts Health NHS Trust v Raqeeb, par. 54.

66 Sentencia citada en la nota 13, par. 99.

67 Ibid., par. 98.

68 Barts Health NHS Trust v Raqeeb, par. 54.
} 
Asimismo, se trajo a colación el art. 21 del TFUE, aunque de forma tímida. El mismo argumento se había formulado por los padres de Evans, que incluso intentaron un habeas corpus, y en ambos casos los tribunales concluyeron que no podía entenderse vulnerado ninguno de esos derechos porque la privación de la libertad lo es por razones terapéuticas ${ }^{69}$.

\section{LA DERIVA CONFESIONAL Y LAS INTROMISIONES INTERNACIONALES}

Los aspectos religiosos están muy presentes en el caso Raqeeb. Así, en distintos párrafos del relato de los hechos se hace hincapié en que la retirada del soporte vital de la niña nunca fue consentida por los padres, principalmente por la fuerza de sus creencias personales y religiosas ${ }^{70}$. Durante la vista, los padres indicaron que la niña había sido iniciada en las prácticas islámicas ${ }^{71}$ y sostuvieron que la decisión del Trust, por haberse hecho de manera totalmente secular, resultaba contraria a la regulación nacional (Equality Act 2010), de la Unión Europea (art. 10 de la Carta de los Derechos Fundamentales de la UE) y del Consejo de Europa (art. 9 del $\mathrm{CEDH})^{72}$. Sostenían igualmente que el mejor interés de su hija tenía que verse en una perspectiva amplia (social, cultural y religiosa) y no en la visión clínica y técnicamente reducida de los médicos ${ }^{73}$.

La posición del Trust fue argumentar que, aunque hubiera implicaciones religiosas, el caso versaba sobre el mejor interés de la niña y esa era la única causa de su decisión y acción ante el tribunal ${ }^{74}$; y que, además, no era su decisión la que en cualquier caso vulneraría la libertad religiosa de la menor, sino su propia situación vital ${ }^{75}$. Respecto a los derechos religiosos de los padres, no tienen, recuerdan, carácter preeminente sobre el interés del menor ${ }^{76}$. En relación con la fatwa, el Trust considera que no resulta de aplicación, pues ni la niña ni sus padres han consentido en la retirada del tratamiento, es el propio Trust el que, a partir de su idea del mejor interés de la menor, busca el parecer del tribunal ${ }^{77}$.

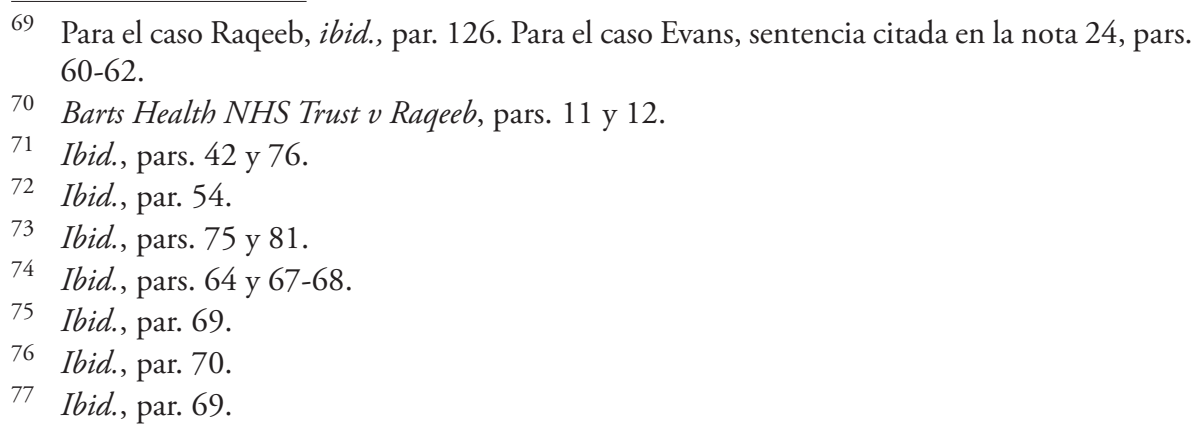


En nombre de la niña, el tutor legal no fue capaz de pronunciarse sobre lo que hubieran sido sus deseos y sentimientos ${ }^{78}$.

En esta situación, el tribunal recuerda que no hay un umbral de edad mínimo para la tenencia del derecho de libertad religiosa, pero que no se trata de un derecho absoluto. Y considera que debe ceder en la ponderación con el mejor interés de la menor, tanto en lo que respecta a su propia libertad religiosa como a la de sus padres ${ }^{79}$. Obiter dicta, el tribunal recuerda además que, por su corta edad, la niña no puede tener una concepción religiosa «sofisticada», aunque acepta que las tradiciones culturales del grupo en el que crece merecen consideración ${ }^{80}$. Respecto a la pretendida discriminación por razones religiosas y la aplicación de la normativa nacional, el tribunal la desestima de plano $^{81}$.

Por otra parte, durante el pleito se recibió un escrito del Alto Comisionado de Bangladesh en el Reino Unido explicando que cualquier ciudadano con esa nacionalidad, incluyendo médicos, que consintieren o participaren en la retirada del tratamiento de la niña serían culpables de un delito penal perseguible ante las instancias de aquel país ${ }^{82}$. Es un aspecto internacional que ha de completarse con las declaraciones obrantes en el expediente por parte del equipo médico enviado por el hospital italiano de Génova, que tomaba como referencia el marco legal y ético de la legislación de ese Estado ${ }^{83}$. En la medida en que la legislación italiana no permite la desconexión en los casos en que no se haya verificado la muerte cerebral, el hospital italiano se mostraba dispuesto a continuar con un tratamiento que, sin embargo, nunca podía llegar a curar a la pequeña ${ }^{84}$. Sí en cambio se esperaba una posible mejora en su calidad de vida, con una traqueotomía que podría llegar a permitir que el resto de los cuidados se le propiciaran en su domicilio.

En el caso Evans, también un hospital italiano se había ofrecido a continuar con el tratamiento del bebé, aunque en aquel caso no existían garantías de una mejora de la calidad de vida. La oferta se realizaba por razones «humanitarias» y rápidamente dio lugar a juicios sociales sobre la presunta barbarie de la postura británica, de las que se hizo eco crítico la doctrina científica ${ }^{85}$.

Ibid., pars. 84 y 85 .

79 Ibid., pars. 129 y 130. En su apoyo se aporta la cita de un precedente de la Corte Suprema Norteamericana, en concreto en el caso Prince v Massachusetts (1944) 321 US 158.

80

81 Ibid., par. 143: «I am likewise not able to accept the submission that in taking the decision it did, the Trust discriminated against Tafida or her parents for the purposes of the Equality Act 2010».

82 Ibid., par. 17.

83 Ibid., par. 38

84 Ibid., pars. 15 y 16

85 C. Barbisan (2018), "Gli sguardi italiani su Alfie Evans», BioLaw Journal - Rivista di Biodiritto, 2, págs. 18-19. 
Dejando al margen las cuestiones extrajurídicas ${ }^{86}$, la injerencia italiana fue mayúscula en el caso Evans con la presunta concesión de una nacionalidad discrecional por carta de naturaleza ${ }^{87}$.

La carta de naturaleza como forma de adquirir la nacionalidad está prevista en Italia en el art. 9.2 de la Ley n. 91, de 5 de febrero de 1992. Tal precepto dispone que, mediante un decreto presidencial, oído el Consejo de Estado y previa deliberación del Consejo de Ministros, a propuesta del Ministerio del Interior, de acuerdo con el ministro de Asuntos Exteriores, la nacionalidad italiana puede ser concedida a un extranjero que haya prestado eminentes servicios a Italia, o cuando concurra en su caso un excepcional interés del Estado ${ }^{88}$. Se pueden predicar de ella las mismas críticas que ha recibido la figura en nuestro ordenamiento jurídico $^{89}$, por discrecional y opaca ${ }^{90}$. Se trata de una figura que descansa sobre

86 Que, sin embargo, han estado tan presentes en los tres casos de referencia que han merecido atención explícita por parte de los tribunales decisores, lo que no es algo usual. Así, Justice Francis, en el caso Gard, hizo hincapié en que las manifestaciones de líderes mundiales relevantes a nivel global, aunque poco familiarizados con los aspectos médicos del caso (sentencia citada en la nota 13, par. 10). Por su parte, Justice Hayden, en el tercero de sus pronunciamientos del caso Evans, condenó expresamente la cantidad de vídeos del pequeño que circulaban por las redes (sentencia citada en la nota 27, par. 2). Finalmente, Justice MacDonald, en el caso Raqeeb, también refiere las urgencias trasladadas por los medios y grupos de presión (sentencia citada en la nota 29, par. 2). Con mayor profundidad sobre este fenómeno puede verse K. McGuire (2019), "Media Framing of "Medical Futility”: Flaming the Debate?», en K. A. Choong (ed.), Medical Futility in Paediatrics: Interdisciplinary and International Perspectives (págs. 95-115), Budapest: Trivent Publishing. Me he ocupado de esta cuestión en el libro editado en 2019, "Citizenship at the Discretion of the State: Public Law Issues Regarding Evans' Naturalisation», en K. A. Choong (ed.), Medical Futility in Paediatrics: Interdisciplinary and International Perspectives (págs. 143162), Budapest: Trivent Publishing.

88 Literalmente: «Con decreto del Presidente della Repubblica, sentito il Consiglio di Stato e previa deliberazione del Consiglio dei Ministri, su proposta del Ministro dell'interno, di concerto con il Ministro degli affari esteri, la cittadinanza puo' essere concessa allo straniero quando questi abbia reso eminenti servizi all'Italia, ovvero quando ricorra un eccezionale interesse dello Stato».

89 Art. 21.1 del Código Civil: La nacionalidad española se adquiere por carta de naturaleza, otorgada discrecionalmente mediante Real Decreto, cuando en el interesado concurran circunstancias excepcionales.

90 La carta de naturaleza es vista por algunos como un atentado a la separación de poderes e incluso al Estado de derecho, mientras que hay quien cree que se puede tratar de una ocasión para realizar justicia material. Desde el punto de vista formal, la determinación de si estamos ante un acto administrativo discrecional, un acto político o un acto graciable aún no ha sido zanjada. Al respecto pueden verse las opiniones contrapuestas de T. Hualde Manso (2011), "Concesión de la nacionalidad por carta de naturaleza. Una institución y una práctica discutibles», Revista Aranzadi Civil-Mercantil, 9, págs. 33-35 y A. Álvarez Rodríguez (2012), «Espańoles por carta de naturaleza: del privilegio a la reparación de 
conceptos jurídicos indeterminados y que, quizá precisamente por ello, muy pocas veces ha sido revisada en sede jurisdiccional ${ }^{91}$.

A mayores, a diferencia de lo que ocurre en España con esta institución, las naturalizaciones italianas no se publican en ningún boletín oficial. Por eso el hecho de que la concesión de la nacionalidad italiana a Alfie Evans nunca fuera oficialmente publicada no puede considerarse equivalente a su no existencia. Ocurre, sin embargo, que la regulación italiana es, como se ha visto, muy meticulosa en los aspectos procedimentales, y que estos no fueron debidamente seguidos en el caso del bebé británico. Así, los primeros ecos respecto a la concesión de la ciudadanía al bebé Evans saltaron a la luz pública tras una declaración pública del ministro italiano de Asuntos Exteriores el 23 de abril de $2018^{92}$. El comunicado de prensa da como un hecho la concesión de la ciudadanía, y la atribuye al referido ministro junto al del Interior, aunque, como ya se ha indicado, estos sujetos no gozan de la capacidad decisoria, sino tan solo de la de propuesta. Al día siguiente, el resumen oficial de actividades del Consejo de Ministros da cuenta de una deliberación de 4 minutos de duración sobre el tema ${ }^{93}$, y la página web oficial de la Presidencia de la República indica que durante esa semana se habría firmado el decreto con la concesión de la ciudadanía ${ }^{94}$. No hay ni rastro empero del preceptivo dictamen previo del Consejo de Estado.

Según la referida nota de prensa del Consejo de Ministros, además, la concesión de la nacionalidad se habría producido en atención al excepcional interés para la comunidad nacional en asegurar al menor la continuidad del tratamiento terapéutico, por la tutela de valores humanitarios preminentes, relacionados con la salvaguarda de la salud ${ }^{95}$. La motivación, si así pudiera verse, no encaja del todo

los perjuicios causados», La Notaría, 3, págs. 42-43. Sobre el difícil control judicial en la materia puede verse el trabajo de A. Hernández de la Torre Navarro (2007), «La vía contencioso-administrativa en la concesión o denegación de la nacionalidad por residencia. Con referencia a la adquisición por carta de naturaleza», en J. M. Bento Company (ed.), Problemas actuales del Registro Civil (págs. 167-210), Madrid: Consejo General del Poder Judicial.

91 Respecto a la situación en Italia puede verse el trabajo de P. Morozzo della Rocca (2008), «La naturalizzazione dello straniero", en P. Morozzo della Rocca (ed.), Immigrazione e cittadinanza. Profili normativi e orientamienti giurisprudenziali (págs. 367-372), Turin: Utet Giuridica. Entre nosotros: A. Álvarez Rodríguez (2008), Nacionalidad española. Normativa e interpretación jurisprudencial, Cizur Menor: Thomson-Aranzadi (en particular págs. 104-108).

92 Cittadinanza Italiana ad Alfie. Disponible en https://www.esteri.it/mae/it/sala_stampa/ archivionotizie/comunicati/2018/04/cittadinanza-italiana-ad-alfie.html.

93 http://www.infoparlamento.it/tematiche/CdM/consiglio-dei-ministri-del-24-aprile-2018-comunicato-stampa.

94 https://www.quirinale.it/page/2018_m04d23.

95 En el original: «Il Consiglio dei Ministri, su proposta del Ministro dell'interno Marco Minniti, ai sensi dell'art. 9, comma 2, della legge 5 febbraio 1992, n. 91, ha deliberato il conferimento della cittadinanza italiana ad Alfie EVANS, nato a Liverpool (Gran Bretagna) 
con la previsión de la legalidad aplicable, pues el tenor del art. 9.2 de la norma de referencia da a entender que la naturalización es, o bien un premio (por eminentes servicios a Italia del beneficiario), o bien un acuerdo de mutuo beneficio (por interés excepcional del Estado ${ }^{96}$ en hacer del beneficiario un nacional); ninguna de tales causas aparece nítidamente en el caso Evans. La referencia a las cuestiones humanitarias suscita además perplejidad en términos de equidad ${ }^{97}$ y sostenibili$\mathrm{dad}^{98}$, teniendo en cuenta que la nacionalidad siempre suele ser vista como un fundamento para la exclusión, y no para la inclusión.

Por si lo anterior no fuera suficiente indicio de que la nacionalidad pudo no haberse conferido de manera válida, resulta que la regulación italiana exige una aceptación recepticia de la nacionalidad conferida, con la debida prestación de un juramento de lealtad a la República y de observancia de la Ley y la Constitución ${ }^{99}$. En España algo así también existe, pero se prevé la dispensa del juramento a los menores de 14 años $^{100}$; no hay tal previsión en la regulación transalpina, por lo que resulta evidente que la nacionalidad italiana nunca llegó a ser efectivamente adquirida por el bebé ${ }^{101}$.

El proceder del Gobierno italiano resulta criticable, además, por contextualmente inoportuno. Se habían producido en aquel tiempo elecciones generales y el Gobierno interino tenía competencias únicamente para asuntos ordinarios, que

il 9 maggio 2016, in considerazione dell'eccezionale interesse per la Comunità nazionale ad assicurare al minore ulteriori sviluppi terapeutici, nella tutela di preminenti valori umanitari che, nel caso di specie, attengono alla salvaguardia della salute».

96 Sujeto que, por cierto, tampoco resulta plenamente identificable con la «comunidad nacional» a que se hace referencia en el acuerdo del Consejo de Ministros.

97 E. Lamarque (2018), "Alfie Evans cittadino italiano. Bene, certamente. Ma perchè solo lui?», BioLaw Journal - Rivista di Biodiritto, 2, págs. 43-44.

98 P. Lombardi (2018), «Giudice amministrativo e cittadinanza: quale contributo per un concetto giuridicamente sostenibile?», Federalismi.it, 20, págs. 2-21 (en particular pág. 13).

99 En un plazo de seis meses, de conformidad con lo previsto en el art. 10 de la Ley italiana de 1992 ya citada, que resulta de aplicación a distintas formas de adquisición de la nacionalidad.

100 J. M. Lete del Río (1996), «Adquisición de la nacionalidad por otorgamiento de carta de naturaleza", Actualidad Civil, 18, págs. 309-414 (en particular pág. 409).

101 En realidad nunca podría haberlo sido, ni en caso de supervivencia, por la imposibilidad de prestar juramento a tan corta edad. La cuestión, posible fuente de discriminación no solo con los menores, sino también respecto a personas con ciertos tipos de discapacidad, ha sido abordada de manera reciente por la Corte Constitucional italiana, y al respecto merece la pena remitirse a las crónicas de S. Rossi (2017), «Incapacitazione e acquisto della cittadinanza. Nota a prima lettura a Corte cost. n. 258/2017", Forum di Quaderni Costituzionali, 12; C. Domenicali (2018), «La "doppia inclusione” dello straniero disabile (a margine di Corte cost. n. 258 del 2017)", Forum di Quaderni Costituzionali, 3 y P. Addis (2018), «Disabilità e giuramento per l'acquisizione della cittadinanza», Consulta Online, II, págs. 435-446. 
la maniobra descrita parece sobrepasar con creces ${ }^{102}$. Finalmente, su movimiento es, desde un punto de vista jurídico, completamente vacuo, pues el cambio de nacionalidad del bebé nada hubiera cambiado en materia de derecho aplicable: la jurisdicción competente es, como se ha visto, la del país de residencia, y ser británico o italiano en nada afectaba a los derechos derivados de la ciudadanía de la Unión. Tampoco la vía diplomática hubiera podido funcionar, pues el obstáculo para el traslado del menor no era una decisión política, sino judicial ${ }^{103}$.

\section{DE NUEVO SOBRE EL ORDEN DE FACTORES: LA LIBRE PRESTACIÓN DE SERVICIOS EN LA UE}

En su acción en el judicial review, los padres de Tafida Raqeeb argumentaban que la decisión del Trust vulneraba el art. 56 del TFUE, pues el corolario de la libre prestación de servicios es la libertad de recibir tales servicios en cualquier Estado miembro ${ }^{104}$. Que los servicios sanitarios entran dentro del ámbito de aplicación del art. 56 queda fuera de toda duda con la mera invocación de la Directiva 2011/24/UE del Parlamento Europeo y del Consejo, de 9 de marzo de 2011, relativa a la aplicación de los derechos de los pacientes en la asistencia sanitaria transfronteriza.

El argumento había sido tímidamente invocado por los padres de Evans en su último intento ante la Corte de Apelación, pero no llegó a ser plenamente discutido por versar aquellos autos solo sobre la admisión a trámite del recurso ${ }^{105}$. También había surgido en alguna fase anterior, pero sin entrar en el fondo, se había zanjado la cuestión recordando sin más que el derecho del art. 56 no es un derecho ilimitado, tratándolo de manera conjunta a la libertad de movimientos y los derechos de los padres ${ }^{106}$.

Resulta bien sabido que los derechos del capítulo segundo del título IV del TFUE, donde se inserta el art. 56, deben ser leídos teniendo en cuenta la posible justificación de las medidas que pudieran vulnerarlos por las razones de interés general que se recogen en el art. 52 del mismo cuerpo normativo. Tales razones son de orden público, seguridad y salud públicas. Para el caso en que pudiera pensarse que la decisión del Trust quedaba cubierta por estas excepcio-

102 A. Ianni (2018), «La concessione della cittadinanza italiana ad Alfie Evans: "Best interests of the child" or "Best interests of the Government?" ", BioLaw Journal - Rivista di Biodiritto, 2, págs. 37-38.

103 De hecho, en el último pronunciamiento del caso, la Corte de Apelación recuerda que el hecho de que el plan de traslado tuviera el apoyo del Papa o del Gobierno italiano nada aporta al interés del menor (sentencia citada en la nota 28 , par. 28).

104 Barts Health NHS Trust v Raqeeb, par. 47.

105 Pronunciamiento citado en la nota 28.

106 Así puede verse en la sentencia citada en la nota 24, par. 53. 
nes, los padres de la nińa hacían valer que no debería entenderse como tal la pendencia del juicio sobre la determinación del mejor interés de su hija, por razones vinculadas a la primacía del derecho de la Unión, porque tal causa no encaja en las «razones imperiosas de interés general» del cdo. 11 de la Directiva 2011/24, y porque ello implicaría imponer a los padres la obligación de agotar los procedimientos nacionales para poder ejercer un derecho de la ciudadanía de la Unión ${ }^{107}$.

Frente a ello, el tribunal recuerda que el procedimiento británico para la determinación del mejor interés del menor tiene una base nacional de residencia de acuerdo con la propia normativa de la Unión (Bruselas II.bis), por lo que no puede admitirse un reparo fundado en un posible conflicto con ella por primacía ${ }^{108}$. En su análisis del procedimiento, concluyó además que, por su causa, la interferencia en el derecho del art. 56 del TFUE podía considerarse justificada por razones de interés público, lo que convierte la actuación del Trust al plantear los autos ante la jurisdicción civil en consistente y no incompatible con el derecho de la UE ${ }^{109}$.

Tales razones de interés público son la protección del mejor interés del niño en el contexto de la decisiones médicas; el interés público en que sean los tribunales quienes resuelvan la disputa entre médicos y familia en estos casos; el interés público en que los facultativos dispongan de un cauce legal para saber lo que el ordenamiento les exige ante un conflicto de este tipo; el interés público en darle una voz independiente al menor en la disputa, y el interés público en asegurar un tratamiento equitativo para todos los niños en la misma situación dentro de la jurisdicción nacional ${ }^{110}$. Estos intereses se materializan en un procedimiento proporcional en objeto y duración y, por lo tanto, puede concluirse, aunque el tribunal evita decirlo expresamente, que la vulneración del art. 56 del TFUE queda justificada en el marco del art. 52 mientras se tramita el procedimiento civil sobre la determinación del mejor interés de la niña ${ }^{111}$.

Entre sus argumentos, la familia había alegado que, de no ser el traslado de la niña contrario a sus intereses, podría primero autorizarse el viaje y después decidirse sobre la cuestión civil. La argucia es descartada por el tribunal, por entender, aunque sin dar mayor explicación, que tal proceder sería más restrictivo para

107 Barts Health NHS Trust $v$ Raqeeb, par. 53. También se argumentaban cuestiones vinculadas a la discriminación, que ya se han sustanciado más arriba.

108 Ibid., pars. 149 y 152

109 Ibid., par. 150.

110 Ibid., par. 151.

111 Es la conclusión que se desprende del par. 154 de la sentencia de referencia, donde se indica que "whilst the national procedure does constitute a prima facie derogation from the EU rights under Art 56, such derogation is accordingly temporary and lasts only as long as necessary to determine the issue in dispute in accordance with the jurisdictional provisions of EU law». 
la libertad en la prestación de servicios ${ }^{112}$. A mayores, se indica, sería contrario al interés del menor autorizar un traslado cuando precisamente está por determinarse qué sea su mejor interés ${ }^{113}$. Y así, determina que, si tras el procedimiento nacional se concluye que el interés del menor es seguir con el tratamiento, entonces podrá hacerse efectivo el derecho del art. 56 del TFUE. Si no, y dado el carácter primario del interés superior del menor también para el ordenamiento europeo, tal derecho no podrá implementarse ${ }^{114}$.

\section{EL INTERÉS SUPERIOR DEL MENOR Y CUESTIONES FINALES}

Sobre el fondo de la cuestión civil en el caso Raqeeb, el tribunal trata de inferir lo que podrían ser los deseos de la menor y analiza la situación de la nińa en cuanto a nivel de consciencia, capacidad para sentir dolor/placer y opciones de tratamiento y cuidados. Es el mismo proceder que se había seguido en los casos Evans y Gard. Cabe destacar además que, también como en aquellos casos, no solo la posición médica se inclinaba hacia la desconexión del soporte vital artificial, también era la opinión del guardián legal conferido para el pleito. Y, sin embargo y a diferencia de los casos previos, en el caso Raqeeb se concluye que el mejor interés de la menor es el mantenimiento del soporte artificial. Sintetizaré seguidamente los elementos más destacados que conducen a esta decisión, siempre teniendo en cuenta la dimensión administrativista ya mencionada.

En respaldo de su opinión, la familia de Raqeeb sostenía que existía acuerdo entre ellos y un cuerpo médico de reputado prestigio (el del hospital italiano) en que sería mejor interés de la niña el mantenimiento del soporte y el traslado, por lo que el tribunal podría incluso llegar a inhibirse. Aportaban como soporte un precedente similar, pero referido a un mayor de edad ${ }^{115}$. El tribunal descarta que tal precedente sea aplicable, no solo por este detalle, sino también porque en aquel caso existía consenso entre la familia y los únicos médicos que trataban al paciente, mientras que en el caso Raqeeb hay una disputa evidente con las autoridades públicas nacionales ${ }^{116}$.

El Tribunal toma su decisión teniendo muy en cuenta que pese a estar moribunda y ser totalmente dependiente, la niña no experimenta dolor, que está médicamente estable y que la carga del tratamiento para mantenerla con vida es bajo, por lo que se inclina por proteger la vida humana. Pondera además la opinión de los médicos italianos sobre la posibilidad de realizar en el futuro intervencio-

112 Además de suponer la subsiguiente falta de jurisdicción de los tribunales británicos por el cambio de residencia.

113 Barts Health NHS Trust $v$ Raqeeb, par. 153.

114 Ibid., par. 154.

115 Sentencia de la Corte Suprema en el caso An NHS Trust $v$ Y [2018] UKSC 4.

116 Barts Health NHS Trust v Raqeeb, pars. 112 y 113. 
nes, como una traqueotomía, que le permitirían continuar el tratamiento en su domicilio, y que el plan de transporte de la menor es seguro y no genera riesgos. Y alcanza su decisión teniendo en cuenta que es la que coincide con la voluntad parental y la que es coherente con el contexto religioso y cultural en que había sido criada y educada la menor.

En la ponderación del tribunal en ningún momento aparece de forma explícita un criterio económico, aunque el hecho de que el plan de tratamiento de la menor estuviera plenamente cubierto desde el punto de vista financiero se destaca en la sentencia en varias ocasiones ${ }^{117}$. La cuestión tiene mucho menor calado personal y humano que la de fondo, pero no es tampoco menor desde la perspectiva de la sostenibilidad de los servicios públicos, en un contexto de recursos económicos limitados. Solo el coste de este tipo de pleitos consume un montante de fondos públicos muy elevado, que se sustrae de la dimensión prestacional ${ }^{118}$. Quizá por ello los representantes legales del Trust habían solicitado durante el pleito que se ofrecieran unas líneas maestras como guía para saber cómo proceder en eventuales casos futuros con derechos derivados de la ciudadanía de la Unión implicados. A este respecto, el tribunal indica que, en la medida en que cada caso tiene sus peculiaridades, no pueden ofrecerse guías detalladas. Con todo, se señala, en el momento presente y ante la demanda de los padres de trasladar al menor a otro Estado miembro para un tratamiento médico, el NHS tendrá en todo caso que considerar los derechos del menor derivados directamente del ordenamiento europeo. Dicho esto, una vez considerados esos derechos, si el NHS entiende que el traslado no se corresponde con el mejor interés del menor y plantea acción a su respecto ante la división familiar del Tribunal Superior, es altamente probable que la decisión constituya una derogación justificada del derecho de la Unión, por razones de orden público, seguridad o salud públicas ${ }^{119}$.

El efecto del fallo en el caso Raqeeb es la continuidad del soporte vital artificial, sea por el NHS, por el Instituto Gaslini en Génova o por otro hospital. Ya no hay razón para interferir en el derecho de la nińa a recibir el servicio sanitario en otro Estado miembro, y por tanto el traslado podrá tener lugar ${ }^{120}$.

117 Siete son las veces en que aparece la expresión fully funded care plan en referencia al plan de traslado y atención en Italia. Las referencias pueden no parecer muchas en una sentencia tan larga, pero se repiten de forma muy concentrada, en el intervalo de los pars. 75-79 y $179-186$.

118 La cuestión ha sido analizada específicamente respecto de los casos Evans y Gard por R. W. M. Law (2019), "Resource-intense Treatments in a Resource-finite Environment», en K. A. Choong (ed.), Medical Futility in Paediatrics: Interdisciplinary and International Perspectives, (págs. 116-142), Budapest: Trivent Publishing. El autor también critica la disparidad de armas que puede surgir cuando un caso se convierte en mediático y la familia recibe un apoyo colaborativo y mediático, desinteresado o no.

119 Barts Health NHS Trust v Raqeeb, par. 189: «[...] it is highly likely that that decision will constitute a justified derogation from the EU rights engaged on public policy grounds».

120 Ibid., par. 188. 
La sentencia se cierra con una serie de citas jurisprudenciales que insisten en la dificultad de tomar decisiones como la de autos. La última de las mismas procede de la Corte Suprema de Estados Unidos ${ }^{121}$, y se emplea para destacar precisamente la relevancia de los contextos públicos y las instituciones administrativas en la determinación del superior interés del niño como «regla de oro» para resolver este tipo de casos ${ }^{122}$. Efectivamente, y como se ha tratado de demostrar, no parece que estas cuestiones puedan ya solventarse únicamente en un ámbito de derecho privado.

${ }^{121}$ En concreto Cruzan v Director, Missouri Department of Health B (1990) 110 S.Ct. 284.

122 Barts Health NHS Trust $v$ Raqeeb, par. 191. 